Int. J. Morphol.,

36(1):113-120, 2018

\title{
Porción Hepática Izquierda (A05.8.01.037)
}

\author{
Left Part of the Liver
}

César Augusto Durand López

\begin{abstract}
DURAND, L. C. A. Porción hepática izquierda (A05.8.01.037). Int. J. Morphol., 36(1):113-120, 2018.
RESUMEN: El conocimiento anatómico es necesario para la cirugía hepática. Los conocimientos acerca de la porción izquierda del hígado que aparecen en los textos de anatomía, cirugía y en la Internet deben ser mejorados y enriquecidos, proponemos hacerlo con este trabajo. Se estudiaron 286 hígados humanos que nos permitieron determinar entre otros conocimientos los siguientes: La porción izquierda del hígado fue más grande que la derecha en $21 \%$ de casos. 2) La porción izquierda del hígado estuvo formada por los cuatro primeros segmentos portales (79 $\%)$ o por los cinco primeros (21\%). 3) La fisura umbilical se inclinó 50 grados hacia la izquierda en relación al plano sagital del hígado y no contuvo a la vena hepática izquierda en $100 \%$ de casos. 4) La vena hepática izquierda tuvo un trayecto intrasegmentario y presentó tres modalidades de drenaje. 5) La irrigación arterial de los segmentos izquierdos I y IV nació de la rama derecha en $16 \%$ y de ambas ramas en $24 \%$. 6) El drenaje biliar de los segmentos derechos VI-VII llegó al conducto hepático izquierdo en $21 \%$ de casos. Estos resultados fueron diferentes de los conocimientos que figuran en los textos de anatomía humana usados en las Escuelas de Medicina a nivel general.
\end{abstract}

PALABRAS CLAVE: Porción izquierda del hígado; Conceptos nuevos.

\section{INTRODUCCIÓN}

Las modernas imágenes radiológicas y las frecuentes intervenciones quirúrgicas en el hígado (A05.8.01.001) hacen necesaria una visión actual de la anatomía de este órgano. Desde hace 63 años se repiten, en la literatura médica, los conceptos de Couinaud, estos conceptos dejan muchos vacíos cuando se trata de explicar la vascularización y la segmentación portal hepática (A05.8.01.033). Distinguidos autores han propuesto segmentaciones portales diferentes pero no han tenido mayor impacto debido a que no se basaron en razones anatómicas estructurales, esto es, que cada segmento portal debe tener tres características para ser considerado como tal: 1) Ser irrigado por ramas terminales de la vena porta hepática y de la arteria hepática propia o sus accesorias. 2) Estar separado de los segmentos vecinos por fisuras portales, creadas por la falta de comunicación vascular entre ellos. 3) Formar parte del canal parenquimal de la porta hepática, lugar donde recibirán su pedículo vascular.

\section{MATERIAL Y MÉTODO}

Se tomó la información acerca del hígado, de los textos de anatomía humana usados en nuestras Facultades de Medicina, de los artículos de revistas especializadas y de los sitios web visitados con frecuencia, como base conceptual, para compararla con los hallazgos de nuestro trabajo. Se estudiaron 286 hígados humanos de diferentes edades, razas y sexo, usando las siguientes técnicas: 150 inyectados con acrílico coloreado en la vena porta hepática (A12.3.12.001), arteria hepática propia (A12.2.12.029), conducto hepático común (A05.8.01.061); 50 disecados en fresco; 30 disecados después de fijarlos con formol; 56 reconstrucciones tridimensionales tomográficas.

Usamos Terminologia Anatomica Internacional (Comité Federativo sobre Terminología Anatómica- FIPAT y Sociedad Anatómica Española, 2001). El número identifica cuando un elemento anatómico se menciona por primera vez en el texto, entre paréntesis se ubican los elementos inconstantes y marcando con + aquellos que no están incorporados en la Terminología Anatómica Internacional.

\section{RESULTADOS}

La porción izquierda del hígado estuvo formada en 226 casos (79 \%) por los segmentos portales I, II, III y IV (Fig. 1). En 60 casos (21\%) estuvo formada por los seg- 


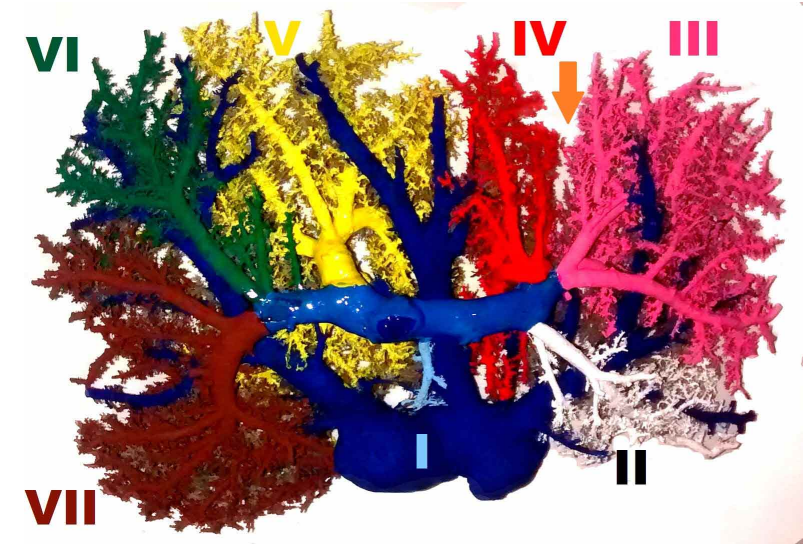

Fig. 1. Cara visceral de un preparado acrílico de hígado, la vena porta y sus ramas primarias en color celeste, las ramas secundarias izquierdas son: Segmentaria I en celeste claro, la II en blanco, la III en rosado, la IV en naranja; las ramas derechas son: Segmentaria $\mathrm{V}$ en amarillo, la VI en verde y la VII en marrón (79 \%). Cuando la rama segmentaria $\mathrm{V}$ nace de la porta izquierda $(21 \%)$ la porción izquierda del hígado es más grande.

mentos I, II, III, IV y V, esto último debido a que la rama portal segmentaria $\mathrm{V}$ (A05.8.01.047) nació de la vena porta izquierda (A12.3.12.005).

Irrigación (Funcional) por la vena porta hepática (A12.3.12.001). Esta vena se dividió en rama derecha (A12.3.12.002) e izquierda (A12.3.12.005) (ramas primarias), luego cada una originó ramas secundarias terminales para cada segmento (Fig. 1), todo esto sucedió en la porta hepatis (A05.8.01.016), elemento anatómico a la vista donde podemos encontrar los pedículos vasculares segmentarios+, estos pedículos se localizaron por debajo de las venas hepáticas de retorno (A12.3.09.005). La vena porta izquierda presentó una porción transversa (A12.3.12.006) y otra anterior umbilical (A12.3.12.008) ambas formaron un ángulo de 90 grados, de seno antero medial. La porción transversa de la porta izquierda presentó una longitud de 3$4 \mathrm{~cm}$, la porción umbilical tuvo $1-2 \mathrm{~cm}$; el diámetro inicial fue de $12 \mathrm{~mm}$. El segmento I (A05.8.01.044) siempre recibió (100\%) una rama (2 mm de diámetro) de la vena porta izquierda y en $60 \%$ además de la vena porta derecha o en 5 $\%$ de la vena porta hepática; el segmento II (A05.8.01.039) siempre (100\%) recibió una rama (4 mm de diámetro) que formó un ángulo de 135 grados de seno posterior con la porción transversa de la vena porta izquierda, esta rama salió desde el ángulo donde se inicia la porción umbilical portal. El segmento III (A05.8.01.040) recibió una rama segmentaria ( $6 \mathrm{~mm}$ de diámetro) y dos o tres subsegmentarias que nacieron a la izquierda del bulbo terminal de la porción umbilical de la vena porta izquierda (10 $\mathrm{mm}$ dediámetro). El segmento IV (A05.8.01.042) recibió una rama segmentaria $(6 \mathrm{~mm})$ que nació a la derecha del bulbo terminal de la porción umbilical de la porta izquierda, además dos o tres ramas subsegmentarias que nacieron a la derecha del citado bulbo o en el dorso de la porción transversa de la vena porta izquierda. En $21 \%$ de casos la rama para el segmento V (10 mm de diámetro) nació de la vena porta izquierda, en $79 \%$ nació de la vena porta derecha, esta rama, luego de profundizarse $2 \mathrm{~cm}$ en su parénquima segmentario dio ramas anteriores, laterales, posteriores y mediales que abarcaron el parénquima comprendido entre la fisura portal derecha (A05.8.01.036) y la fisura portal principal (A05.8.01.035), desde el margen inferior del hígado (A05.8.01.024) hasta la vena cava inferior (A12.3.09.001) por detrás. Esta rama es la única existente para la división medial derecha (A05.8.01.046) o segmento V de nuestra nomenclatura (Durand, 2016, 2017).

Irrigación arterial (nutricional) de la porción izquierda. La irrigación arterial provino de la hepática propia y/o una rama de la gástrica izquierda (A12.2.12.013). La hepática izquierda (A12.2.12.035) fue corta y menos voluminosa que la hepática derecha (A12.2.12.030). Al nacer, la rama derecha y la izquierda se ubicaron por delante de la vena porta izquierda. En $80 \%$ de casos la rama izquierda se ubicó por delante y debajo de la porción transversa de la vena porta izquierda (Fig. 2). La rama izquierda dio una rama hacia atrás para el segmento I, al llegar al ángulo de la vena porta izquierda, dio una rama postero-lateral izquierda la que se ubicó por encima del ángulo portal destinada al segmento II, luego, colocándose medial a la porción umbilical de la vena porta izquierda dio las ramas para el segmento IV y el segmento III, esta última pasó por debajo de la porción umbilical de la vena porta camino a su pedículo segmentario; la rama para el segmento IV se colocó en el margen medial de la porción umbilical de la vena porta izquierda. Observamos que las ramas para los segmentos posteriores cursan

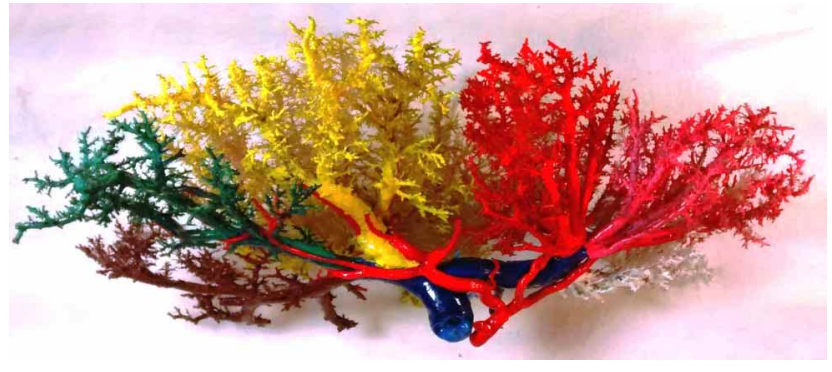

Fig. 2. Vista antero-ventral de un preparado acrílico donde vemos a la arteria hepática propia, a la izquierda de la vena porta, dividirse en una rama derecha grande y larga, y otra izquierda pequeña y corta, de esta se ve nacer las segmentarias IV y III. De la rama derecha nace la segmentaria I, luego la cística, luego la segmentaria V, la VI y la VII. Las ramas primarias se ubican debajo de la vena porta izquierda. Se aprecia la forma cónica de los segmentos portales, cuyos vértices apuntan a la porta hepática. 
por encima de las ramas venosas portales, para los segmentos anteriores cursan por debajo de las ramas venosas portales. El $70 \%$ de hígados estuvo irrigado por la hepática propia únicamente, de este porcentaje el segmento I recibió ramas de la hepática izquierda en $30 \%$, de la derecha en $16 \%$ y de ambas ramas en $24 \%$. El segmento II recibió siempre una rama de la hepática izquierda. El segmento III recibió siempre una rama de la hepática izquierda. El segmento IV recibió una rama de la hepática izquierda en $50 \%$ y de la hepática derecha en $20 \%$. El segmento $\mathrm{V}$, sea su rama portal izquierda o derecha, fue irrigado por una o dos ramas de la hepática derecha en $69 \%$.

Drenaje biliar. Las ramas biliares segmentarias acompañaron a las arteriales y venosas portales hasta que formaron el conducto hepático izquierdo (A05.8.01.065) y el conducto hepático derecho (A05.8.01.062), estos troncos se ubicaron por encima de las ramas venosas portales primarias, al unirse lo hicieron delante y a la derecha de la vena porta en la porta hepatis. Se observó que dos conductos segmentarios, ya sea en el lado derecho o izquierdo, se unieron en un tronco común, el que ligado al tercer o cuarto conducto biliar de la porción correspondiente, formaron el conducto hepático, ya sea derecho o izquierdo. Generalmente, los conductos biliares I, II, III y IV constituyeron el conducto hepático izquierdo y los conductos segmentarios V, VI y VII formaron el conducto hepático derecho. Esta confluencia es más constante en el lado izquierdo. El drenaje del segmento I fue en el conducto izquierdo (70\%), en el derecho (7\%) y en ambos ( $23 \%)$; el tronco II-III en el conducto izquierdo en $76 \%$ y el tronco III-IV en el conducto izquierdo en $24 \%$. El tronco VI-VII del lado derecho desembocó en el conducto izquierdo en $21 \%$ de casos, este no lo hizo más allá de un centímetro del punto de unión de ambos conductos hepáticos, aún en adultos.

Las fisuras portales verticales. Son planos de separación originados por la falta de anastomosis entre las ramas portales segmentarias+, presentaron inclinaciones en relación al plano sagital (imaginario) del hígado. La fisura derecha (A05.8.01.036) se inclinó 60 grados hacia la derecha, la fisura principal (A05.8.01.035) 30 grados hacia la izquierda, la fisura umbilical (A05.8.01.034) 50 grados hacia la izquierda. El contenido de la fisura principal fue la vena hepática intermedia (A12.3.09.007) en $100 \%$ de casos (Fig. 3). La fisura umbilical tuvo en su margen inferior, de adelante hacia atrás al ligamento redondo del hígado (A05.8.01.015), la porción umbilical de la porta izquierda y al ligamento venoso (A05.8.01.011), en el margen superior de esta fisura se implantó el ligamento falciforme del hígado (A10.1.02.303), esta fisura nunca contuvo a la vena hepática izquierda (Fig. 4). En el tercio posterior de la fisura umbilical se vio una pequeña vena (Fig. 5) que drenó el dor-

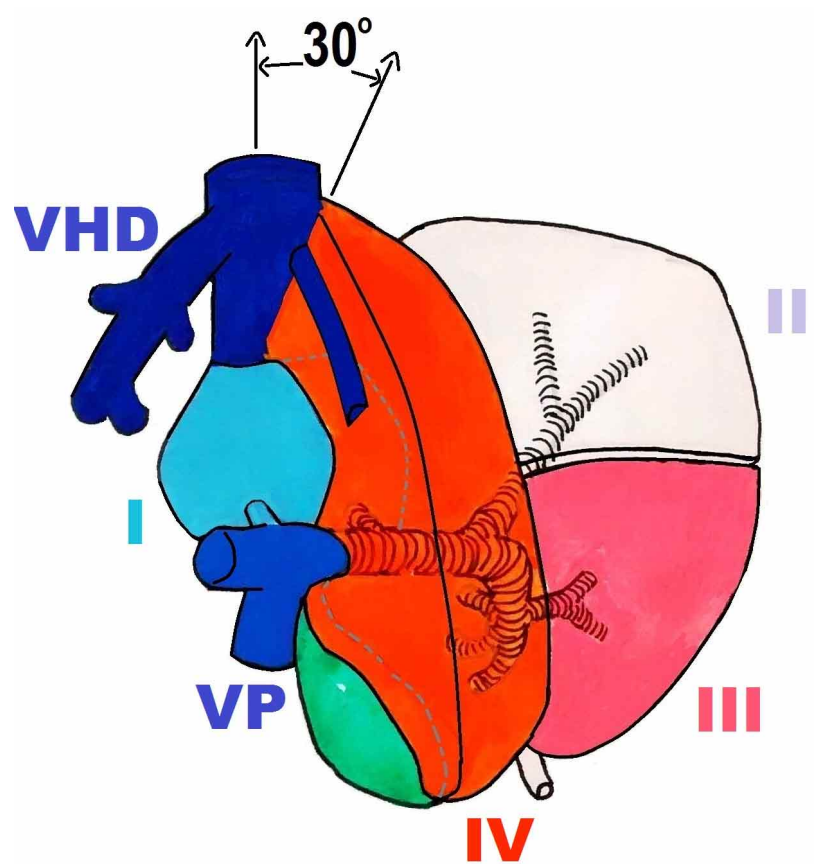

Fig. 3. Esquema de la porción izquierda del hígado con sus segmentos portales. La fisura principal que divide ambas porciones, tiene una inclinación izquierda de 30 grados. Se inicia a lo largo del lecho de la vesícula biliar, pasa sobre la bifurcación portal y luego se ubica sobre la mitad del segmento I. La mitad posterior del segmento IV (naranja) descansa sobre la mitad izquierda del segmento I (celeste). El contenido de la fisura principal es la vena hepática intermedia.

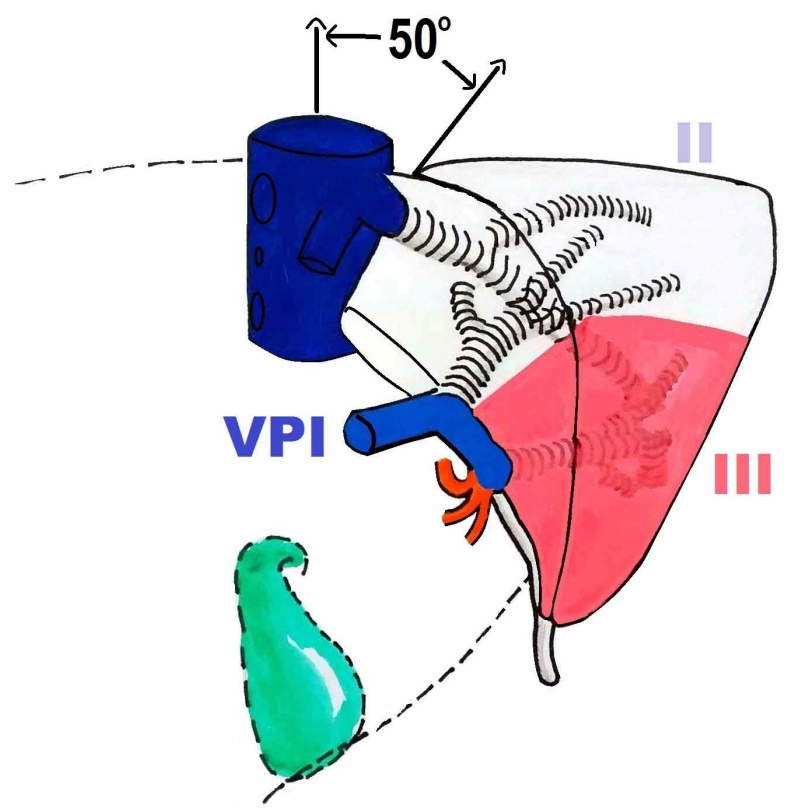

Fig. 4.- Esquema de la fisura umbilical y la división lateral izquierda. La fisura presenta una inclinación izquierda de 50 grados, en su margen inferior, de delante hacia atrás, contiene al ligamento redondo del hígado, a la porción umbilical de la rama portal izquierda y al ligamento venoso; no contiene a la vena hepática izquierda, cuyo trayecto es intrasegmentario (III-II). 
so del segmento IV (50\%) y desembocó en la vena hepática izquierda $(28 \%)$ casi en su desembocadura, en la vena hepática intermedia (15\%) o en la vena cava inferior (7\%). La vena hepática izquierda (A12.3.09.008). Presentó trayecto intrasegmentario (segmentos III-II), con tres modalidades de drenaje, la primera y más frecuente $(80 \%)$ nació en el segmento III, cursó a través del II recibiendo afluentes, hizo una curva de concavidad medial antes de desembocar (Fig. 6). En la segunda modalidad (20\%) cada segmento (III y II) ofreció una vena de drenaje, las cuales se unieron para formar la hepática izquierda (Fig. 7). Independientemente de su formación y drenaje, $28 \%$ de las venas hepáticas izquierdas recibió como última afluente a la vena de la fisura

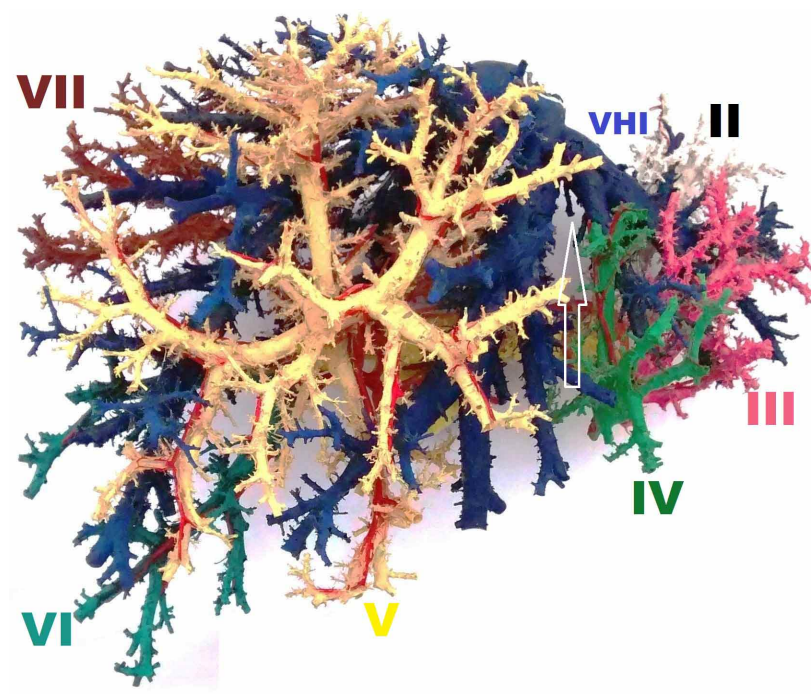

Fig. 5. Vista frontal de un preparado acrílico de hígado, la flecha señala la vena fisural o vena de la fisura umbilical (28\%).

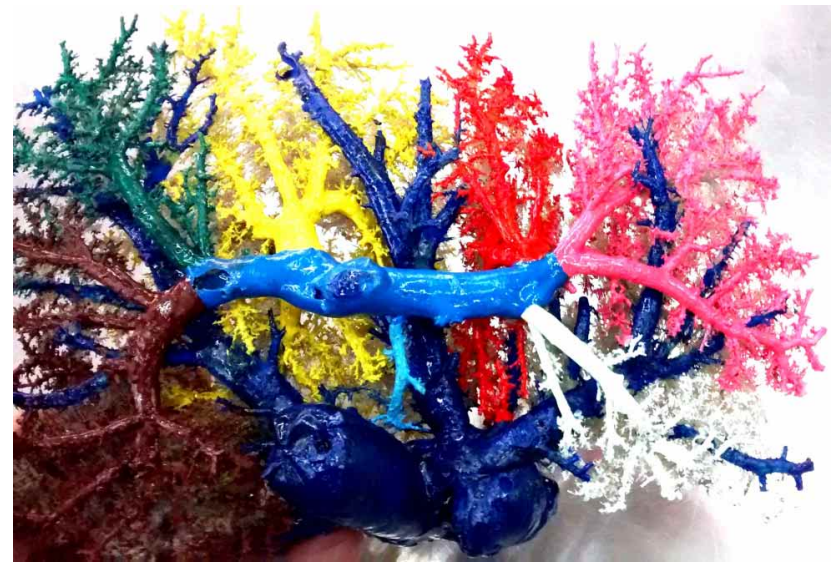

Fig. 6. Vista de la cara visceral de un preparado acrílico de hígado. Se ve nacer la vena hepática izquierda, dentro del parénquima del segmento III (rosado), como un tronco que recibe afluentes, luego ingresa al segmento II (blanco) y continúa recibiendo afluentes, hace una curva de concavidad medial para dirigirse a su desembocadura $(80 \%)$. umbilical (dorso del segmento IV) convirtiéndose en la tercera modalidad (Fig. 5). La vena hepática izquierda desembocó en la vena cava inferior ( $3 \%$ ) o en la vena hepática intermedia $(97 \%)$. La vena hepática intermedia, siempre estuvo presente, recibió afluentes más numerosas y de mayor calibre desde el lado derecho (segmento V) que desde el lado izquierdo (segmento IV).

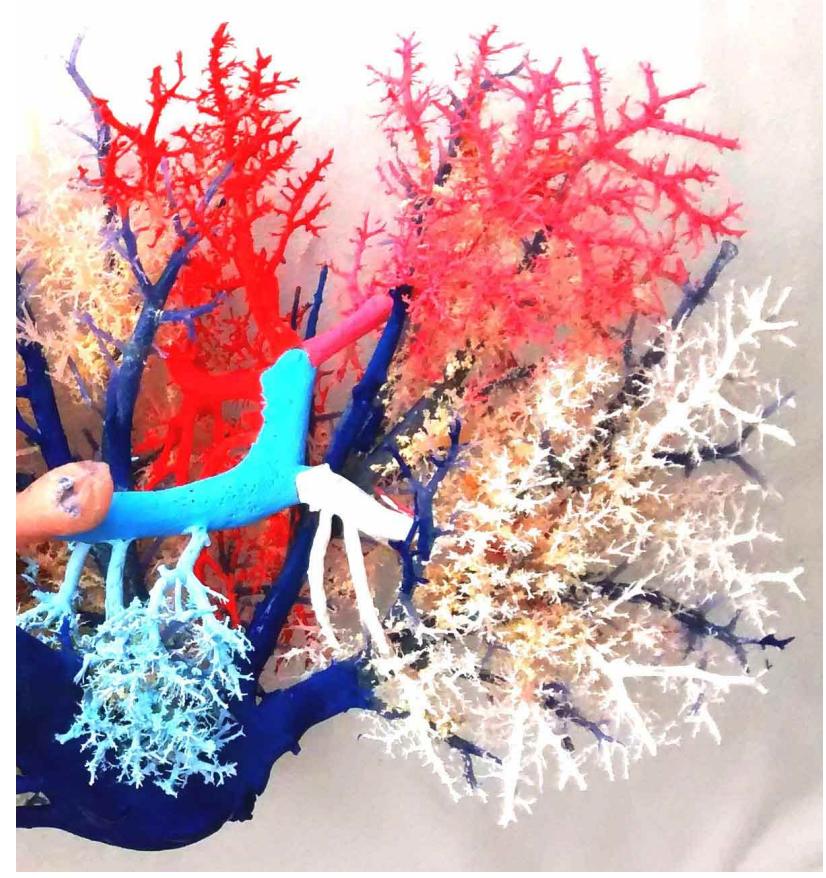

Fig. 7. Vista de la cara visceral de un preparado acrílico de hígado. Se ve nacer la vena hepática izquierda a partir de dos ramas, una que drena el segmento III (rosado) y otra que drena el segmento II (blanco) $(20 \%)$.

\section{DISCUSIÓN}

La denominación "porción izquierda del hígado" (del latín portio, o-nis: Cantidad segregada de otra mayor. Cantidad que corresponde a cada partícipe en un reparto o distribución), refleja el concepto quirúrgico de la división del pedículo vascular inferior y su parénquima correspondiente. La denominación "lóbulo izquierdo del hígado" (A05.8.01.027) (del latín lobulus, y este del griego lob V lobós 'lóbulo' y el sufijo diminutivo latín ulus: Anatomía: Porción redondeada y saliente de un órgano cualquiera) describe la característica externa del hígado. El concepto de porción hepática creció desde la descripción de Cantlie en 1898, de un plano que dividía el hígado en porción derecha e izquierda irrigadas por las ramas de la vena porta hepática y la arteria hepática propia. Por esta razón esta fisura se convirtió en la más importante y se denominó fisura principal 
(A05.8.01.035), sin embargo, cuando encontramos que (Durand 2016, 2017) la vena segmentaria portal V (que irriga toda la división medial derecha) nació de la vena porta izquierda $(21 \%)$ la porción izquierda del hígado resultó mayor que la derecha, siendo la fisura portal derecha la que dividió el hígado en porciones derecha e izquierda, ejerciendo el rol de fisura principal.

Acerca de la irrigación portal del hígado, Standring (2008) escribió: "The left portal vein has a longer extraparenchymal course $(4-5 \mathrm{~cm})$ and tends to lie slightly more horizontally than the right portal vein, but is often of smaller caliber. It has horizontal and vertical portions. The horizontal portion runs along the base of segment VI and often gives branches to segment I and sometimes to segment VI in this part of its course. The branch to segment II continues laterally but the main left portal vein takes a more anterior and vertical course in the umbilical fissure (the vein of the umbilical fissure) where it gives branches to segments III and IV and receives the obliterated left umbilical vein (ligamentum teres). The majority of the supply to segment IV comes from the left portal vein, and only occasionally from the right via proximal branches of the main vein or branches from veins to segments V or VIII". En las nuestras que estudiamos, la porción transversa de la vena porta izquierda no tuvo relación con el segmento VI y nunca le dio una rama (están en extremos opuestos del hígado). La rama para el segmento II fue siempre latero-posterior, formó un ángulo de 135 grados de seno posterior con la porción transversa de la vena porta izquierda. La denominación "vena de la fisura umbilical" no corresponde a la porción umbilical de la vena porta izquierda sino corresponde a una pequeña vena que drena el dorso del segmento IV y desemboca en la vena hepática izquierda ( $28 \%$ ) en el tercio posterior de la fisura umbilical. El segmento IV no recibió ninguna rama de la porta derecha, su pedículo vascular nació muy lejos de la vena porta derecha. Una rama subsegmentaria para el segmento I nació directamente de la rama portal derecha en 60 $\%$ o de la vena porta hepática en $5 \%$ de casos.

Moore et al. (2009) escribieron acerca de la irrigación portal: "En el porta hepático, o cerca, la arteria hepática propia y la vena porta hepática terminan dividiéndose en ramas derecha e izquierda, estas ramas primarias irrigan las porciones hepáticas derecha e izquierda respectivamente. Dentro de cada porción las ramificaciones secundarias simultáneas de la vena porta hepática y de la arteria hepática propia irrigan las divisiones mediales y laterales de las porciones derecha e izquierda del hígado. Tres de las cuatro ramas secundarias sufren una nueva ramificación terciaria para irrigar de manera independiente siete de los ocho segmentos hepáticos". Este tipo de ramificación ubicaría a los segmentos en la periferie del hígado, lejos de la porta hepá- tica. El canal parenquimal de la porta hepática está formado por parte del parénquima de todos y cada uno de los segmentos por ser este, el lugar donde recibirán su pedículo vascular terminal; todas estas ramas mencionadas por Moore et al. tendrían que estar en la porta hepática, sin embargo, ni la porta hepática es tan grande y amplia, ni presenta tal congestión de ramas. En nuestro trabajo las ramas portales primarias derecha e izquierda emitieron, en forma alternada, siete ramas secundarias terminales para siete segmentos, estos siete pedículos vasculares ingresaron a su territorio segmentario correspondiente allí donde el segmento formó parte del canal de la porta hepática. La vena porta izquierda dio las ramas para los segmentos I, II, III y IV y la derecha para los segmentos V, VI y VII en $79 \%$ de casos; en $21 \%$ de casos la vena porta izquierda dio las ramas para los segmentos I, II, III, IV y V y la derecha para los segmentos VI y VII. Además, erróneamente dice Moore et al. que las ramas secundarias son 4 y que tres de ellas se subdividen en 2 para sumar 7 pedículos segmentarios de los 8 que existen. ¿De dónde sale el octavo pedículo? sino lo sacan de una rama periférica del segmento $\mathrm{V}$, no nace en la porta hepática, en los esquemas de la segmentación portal propuesta por Couinaud y repetidas por Moore et al. no vemos que el segmento VIII forme parte del canal de la porta hepática, no hemos visto salir esta rama ni de la vena porta izquierda ni de la derecha.

Latarjet \& Ruiz Liard (2006) describieron la clasificación segmentaria de Couinaud con dibujos de un hígado dividido en segmentos cúbicos, esquemáticos; siempre vemos dibujos de su segmentación, nunca una foto, porque la realidad es distinta, la distribución segmentaria es radiada, centrífuga, nace en la porta hepática y se expande radialmente hacia la periferie, es por esta razón que los segmentos semejan forma de cono truncado e invertido (recuerda las pirámides renales, con vértice interno y base periférica), forma favorecida por la inclinación, concavidades y convexidades de las fisuras portales y las suaves curvas de la superficie hepática. En todos los hígados estudiados encontramos siete pedículos portales segmentarios y algunas pequeñas ramas subsegmentarias, en $79 \%$ de casos los segmentos portales izquierdos fueron los cuatro primeros, en $21 \%$ fueron los cinco primeros, siendo el pedículo $\mathrm{V}$ el que varió su origen.

Acerca de la irrigación arterial del hígado, Standring mencionó "Collateral circulation occurs between segments via fine terminal branches"... "The segment VI artery most commonly arises from the left hepatic artery, but in about $10-20 \%$ of cases it arises from the right hepatic artery or the main hepatic artery". Standring se equivoca al decir que hay circulación colateral entre los vasos terminales, primero porque no la hay y segundo porque al decir terminal expresa que tiene un final sin posibilidad de anastomosis. Cuan- 
do dice que la rama segmentaria VI nace comúnmente de la rama izquierda se equivoca, en ninguna otra bibliografía se describe lo que dice; en nuestra investigación la arteria segmentaria VI nació siempre (100 \%) de la rama derecha. Moore et al. no describen la irrigación arterial segmentaria. Latarjet \& Ruiz Liard describieron: "Las ramas terminales penetran en el porta hepático, además de la rama correspondiente de la vena porta hepática, y se expanden en el hígado, siguiendo las ramificaciones venosas. La rama derecha es más voluminosa que la izquierda. Sus ramas arteriales son anatómica y funcionalmente terminales". Concepto contrario a lo escrito en Gray's anatomy acerca de las anastomosis intersegmentarias. En nuestros hígados la irrigación arterial fue mediante la hepática propia en $70 \%$ de casos, la hepática propia y una rama de la gástrica izquierda en $25 \%$, la hepática propia más una rama de la gástrica izquierda más una rama de la mesentérica superior en $3 \%$ y por la hepática propia y una rama de la mesentérica superior en $2 \%$, en cada caso las ramas participantes se anastomosaron en la porta hepatis antes de ingresar a su segmento correspondiente como arterias terminales. La rama derecha siempre fue más larga y voluminosa que la izquierda. Cuando solo la hepática propia irrigó el hígado (70 \%), la rama segmentaria I nació de la rama izquierda en $30 \%$, de la derecha en $16 \%$, y de ambas ramas en $24 \%$. La rama segmentaria II nació de la rama izquierda en todos los casos. La rama segmentaria III nació de la rama izquierda en todos los casos. La rama segmentaria IV nació de la rama izquierda en $50 \%$ y de la derecha en $20 \%$ variación descrita también por Jin et al. (2008). Panagouli et al. (2011) reportaron que las arterias hepáticas accesorias pueden nacer de la gástrica izquierda o la mesentérica superior, para ambos lados, solo el ingreso a su territorio correspondiente las numera y clasifica. Generalmente, las arterias se situaron por debajo de los conductos biliares hepáticos. En el $80 \%$ de casos la rama izquierda se ubicó debajo de la porta izquierda y en $20 \%$ sobre ésta.

Los textos de Gray, Moore et al. y Latarjet \& Ruiz Liard no mencionan la formación del drenaje biliar. Mitidieri \& Mitidieri (2017) describieron las formas de drenaje biliar de la porción izquierda del hígado, Mitidieri \& Ottone (2015) informaron los porcentajes de las formas de drenaje descritas en 83 casos. En nuestra casuística los conductos biliares de los segmentos portales I, II, III y IV constituyeron el conducto hepático izquierdo y los conductos segmentarios V, VI y VII formaron el derecho. Esta confluencia fue más constante en el lado izquierdo. El segmento I drenó en el hepático izquierdo en $70 \%$, en el derecho en $7 \%$, en ambos conductos en $23 \%$; el hepático izquierdo se formó por el tronco de los segmentos II-III en $76 \%$ y los conductos III-IV en 24 $\%$. La variación más frecuente fue el drenaje del tronco VIVII (derechos) en el conducto izquierdo en $21 \%$, el hepáti- co común en $6 \%$ y en el cístico en $2 \%$. Esta desembocadura nunca fue más allá de $1 \mathrm{~cm}$ del nacimiento del hepático común.

Standring describió tres conceptos acerca de la fisura de la porción izquierda: la fisura umbilical, la fisura venosa y la fisura portal izquierda, como entidades diferentes, diferente ubicación y diferente contenido, de una manera confusa que se contradice en cada definición: 1) "Left portal fissure: It extends from the mid point of the anterior edge of the liver between the falciform ligament and the left triangular ligament to the point which marks the confluence of the left and middle hepatic veins. It contains the left hepatic vein and separates the left anterior and left posterior sectors"... 2) "The umbilical fissure separates segment III from segment VI within the left anterior sector and contains a main branch of the left hepatic vein (the umbilical fissure vein). It is marked by the attachment of the falciform ligament and sometimes covered by a ridge of liver tissue extending between the segments: it is often avascular and can be divided safely"... 3) "The venous fissure is a continuation of the umbilical fissure on the under surface of the liver and contains the ligamentum venosum. It lies between the caudate lobe and segment IV. The deeper continuation of this plane is the dorsal fissure".

Corregimos la primera afirmación de Standring diciendo que su fisura portal izquierda está ubicada entre el segmento portal IV y los segmentos III-II, va desde la incisura del ligamento redondo en el margen inferior del hígado hasta el margen izquierdo de la vena cava inferior por detrás, no contiene a la vena hepática izquierda. Corregimos la segunda afirmación de Standring recordando que la fisura umbilical separa el segmento IV de los segmentos II-III en la cara diafragmática; por la cara visceral separa el segmento III del IV por delante y el segmento I del II por detrás. En la zanja que forma la fisura umbilical en la cara visceral tenemos por delante al ligamento redondo del hígado, esta porción fisural se conoce como fisura del ligamento redondo, luego le sigue la porción umbilical de la vena porta izquierda y finalmente por detrás, el ligamento venoso en la fisura del ligamento venoso, la fisura umbilical tiene una inclinación izquierda de 50 grados en relación con el plano sagital del hígado. Corregimos la tercera afirmación recordando que el lugar de la fisura umbilical que aloja al ligamento venoso, separa el segmento I del II; la mitad posterior del segmento IV descansa sobre la mitad izquierda del segmento I.

Moore et al. describieron las fisuras hepáticas como fisuras sagitales. Latarjet \& Ruiz Liard escribieron acerca de la inclinación del plano de la fisura umbilical "La vena hepática izquierda drena la sangre del lóbulo izquierdo a 
partir de dos raíces: Una transversal posterior (segmento II) y otra anteroposterior (segmento III): está situada en un plano sagital suprayacente a la fisura del ligamento redondo y al conducto venoso del hígado; es la fisura umbilical". Estas afirmaciones difieren con nuestros resultados y la descripción de (Sinnatamby, 2003) en la que el plano de la fisura umbilical se inclinó 50 grados hacia la izquierda. Nosotros encontramos que la fisura portal izquierda y la fisura umbilical son denominaciones de un mismo elemento anatómico, esta contiene en el margen inferior a la fisura del ligamento redondo y a la fisura del ligamento venoso.

Cuando la rama segmentaria $\mathrm{V}$ nació de la vena porta derecha, el plano de división entre las porciones derecha e izquierda del hígado fue la fisura portal principal, cuando la rama segmentaria $\mathrm{V}$ nació de la vena porta izquierda el plano de división fue la fisura derecha.

El ligamento falciforme se implantó sobre la fisura umbilical, entre la división lateral y medial de la porción izquierda del hígado, a lo largo de esta fisura el parénquima tuvo un grosor, desde el fondo de la fisura en la cara visceral hasta la cara diafragmática de: $5-10 \mathrm{~mm}$ en la fisura para el ligamento redondo, $15-20 \mathrm{~mm}$ a nivel del bulbo terminal de la porción umbilical de la porta izquierda, $100 \mathrm{~mm}$ a nivel del ángulo de la porta izquierda y de $100-120 \mathrm{~mm}$ a nivel del margen izquierdo de la cava inferior.

En relación a la ubicación de la vena hepática izquierda: (Standring 2008) escribió: "The left hepatic vein lies between the left medial and left lateral sectors of the liver". Moore et al. escribieron: "Las porciones derecha e izquierda del hígado se subdividen, por un plano vertical, en divisiones medial y lateral por las fisuras portales derecha y la izquierda (umbilical), en las que se ubican las venas hepáticas derecha e izquierda". Latarjet \& Ruiz Liard ubicaron la vena hepática izquierda en la fisura umbilical. Flores Rivera (2007) escribió: "La cisura portal izquierda contiene la vena suprahepática izquierda y separa al hígado izquierdo en dos sectores, anterior y posterior". Micheau (2017) describió: "The umbilical fissure runs parallel to the left side of the falciform ligament, following behind the fissures for the round ligament. The umbilical fissure separates the medial and lateral segments forming a boundary for hepatic segmentation (projection on the surface of the liver of the left hepatic vein)". Nuestros hallazgos demuestran que la vena hepática izquierda tiene trayecto intraparenquimal a través de los segmentos III y II y no se ubica en la fisura umbilical sino que la atraviesa inmediatamente antes de su desembocadura.

Acerca de la formación, afluentes y desembocadura de la vena hepática izquierda. (Standing 2008) escribió que la vena hepática izquierda drena los segmentos II, III y ocasionalmente IV mediante la vena de la fisura umbilical. Moore et al. no describieron la vena hepática izquierda. Latarjet \& Ruiz Liard describieron que la vena hepática izquierda drena la sangre del lóbulo izquierdo a partir de dos raíces: Una transversal posterior (segmento II) y otra anteroposterior (Segmento III)". Egawa (1997) y Reichert et al. (2000) describieron al igual que Gomes da Silveira \& Ribeiro Filho (2017) los tipos de drenaje de la vena hepática izquierda. Dar et al. (2008) reportó el drenaje por separado del segmento II y del III en la vena cava inferior. Fang et al. (2012) describió que el patrón prevalente del drenaje de la hepática izquierda fue en la vena hepática intermedia (122/200, $61 \%)$, también encontró que el dorso del segmento IV drenó en la hepática izquierda en $26 \%$ de casos, en la vena hepática intermedia en $17,5 \%$, o en la vena cava inferior en $8 \%$. Kimura et al. (2015) describieron la formación de un tronco común de desembocadura de la vena hepática intermedia y la izquierda que midió 0,2-1,7 cm de largo en $84 \%$ de un total de 83 casos. Asimismo describieron la vena de la fisura umbilical entre la vena hepática izquierda y la intermedia. Según Couinaud (1989), la vena de la fisura umbilical se presentó en 51,5\% de 97 casos. En nuestra experiencia la "vena fisural" discurrió en el tercio posterior de la fisura umbilical. Sano et al. (2003) reportaron la vena de la fisura umbilical en $81 \%$ de 21 casos. Tani et al. (2016) describieron que la vena hepática izquierda, la intermedia y la derecha drenan en promedio $20,7 \%, 32,7 \%$, y 39,6 \% del parénquima hepático, respectivamente, las venas accesorias drenan el $7 \%$ restante. La vena hepática derecha media e inferior drenan el $8 \%$ y $10,6 \%$, respectivamente. La vena de la fisura umbilical se observó en $96 \%$ con un diámetro de $>3 \mathrm{~mm}$, esta vena drenó en la hepática izquierda en $87,2 \%$, en la vena intermedia en $7,3 \%$.

Nosotros observamos tres tipos de drenaje de la vena hepática izquierda: 1) Un tronco común (80\%) que nació en el centro del segmento III, recibió sus tributarias, ingresó al segmento II atravesando perpendicularmente la fisura horizontal entre los segmentos II y III, formó una curva de concavidad medial, recibió tributarias del segmento II, cruzó el plano de la fisura umbilical para desembocar en la Vena Cava Inferior o en la Vena Hepática Intermedia. 2) Dos ramas segmentarias separadas, una rama del segmento III más una rama del segmento II que formaron la Vena Hepática Izquierda (20\%). 3) Cualquiera de las variantes anteriores recibiendo la vena del dorso del segmento IV o vena fisural $(28 \%)$. En nuestros casos la Vena Hepática Izquierda desembocó en la Vena Hepática Intermedia (97 $\%)$ para formar un tronco común, o en la Vena Cava Inferior en $(3 \%)$. La vena fisural $(28 \%)$ se ubicó entre los segmentos II y IV, en el tercio posterior de la fisura umbilical. 
DURAND, L. C. A. Left Part of the Liver. Int. J. Morphol., 36(1):113-120, 2018.

SUMMARY: Accurate anatomical knowledge is necessary for liver surgery. Much of the knowledge about the left portion of the liver in the anatomy, surgery and internet texts must be improved and enriched; we propose to do this with this work. We studied 286 human livers that allowed us to determine further knowledge, along with the following information: 1) The left liver portion was larger than the right liver in $21 \%$ of cases. 2) The left portion of the liver was formed by the first four portal segments (79\%) or by the first five $(21 \%)$. 3) The umbilical fissure tilted 50 degrees to the left relative to the sagittal plane of the liver and did not contain the left hepatic vein in $100 \%$ of cases. 4) The left hepatic vein had an intrasegmental path and presented three drainage modalities. 5) Arterial irrigation of the left segments I and IV were born from the right branch in $16 \%$ and from both branches in $24 \%$. 6) Bile drainage of right segments VI-VII reached the left hepatic canal in $21 \%$ of cases. These results were different from the knowledge contained in the human anatomy texts used in medical schools at the general level.

KEY WORDS: Left portion of the liver; New concepts.

\section{REFERENCIAS BIBLIOGRAFICAS}

Comité Federativo sobre Terminología Anatómica \& Sociedad Anatómica Española. Terminologia Anatomica: Terminología Anatómica Internacional. Madrid, Médica Panamericana, 2001.

Couinaud, C. Surgical Anatomy of the Liver Revisited. Paris, C. Couinaud, 1989.

Dar, F. S.; Faraj, W.; Heaton, N. D. \& Rela, M. Variation in the venous drainage of left lateral segment liver graft requiring reconstruction of segment III vein with donor iliac artery. Liver Transpl., 14(4):576-9, 2008.

Durand, L. C. A. Liver: Seven Hepatic Segments. Int. J. Morphol., 34(4):1522-30, 2016

Durand, L. C. A. Liver: the portal segment V. Int. J. Morphol., 35(1):3637, 2017.

Egawa, H.; Inomata, Y.; Uemoto, S.; Asonuma, K.; Kiuchi, T.; Okajima, H.; Yamaoka, Y. \& Tanaka, K. Hepatic vein reconstruction in 152 living-related donor liver transplantation patients. Surgery, 121(3):2507, 1997.

Fang, C. H.; You, J. H.; Lau, W. Y.; Lai, E. C.; Fan, Y. F.; Zhong, S. Z.; Li, K. X.; Chen, Z. X.; Su, Z. H. \& Bao, S. S. Anatomical variations of hepatic veins: three-dimensional computed tomography scans of 200 subjects. World J. Surg., 36(1):120-4, 2012.

Flores Rivera, E. Anatomía del Hígado y de las Vías Biliares. San José de Costa Rica, Cirugía Hepatobiliar y Transplante Hepático, 2007. Disponible en: https://drfloresrivera.wordpress.com/2007/09/11/anatomiadel-higado-y-de-las-vias-biliares.

Gomes da Silveira, V. \& Ribeiro Filho, J. Anatomía y Fisiología Hepática. Sao Paulo, Cirugía Sanchinarro, 2017. Disponible en: http:// www.cirugiasanchinarro.com/sites/default/files/gonzales02.pdf

Jin, G. Y.; Yu, H. C.; Lim, H. S.; Moon, J. I.; Lee, J. H.; Chung, J. W. \& Cho, B. H. Anatomical variations of the origin of the segment 4 hepatic artery and their clinical implications. Liver Transpl., 14(8):1180-4, 2008.

Kimura, W.; Fukumoto, T.; Watanabe, T. \& Hirai, I. Variations in portal and hepatic vein branching of the liver. Yamagata Med. J., 33(2):11521, 2015.
Latarjet, M. \& Ruiz Liard, A. Anatomía Humana. Tomo 2. Madrid, Médica Panamericana, 2006.

Micheau, A. Fisura Umbilical. Montpellier, IMAIOS: sitios web de formación electrónica e información médica para los profesionales sanitarios, 2017. Disponible en: https://www.imaios.com/es/e-Anatomy/Estructuras-anatomicas/Fisura-umbilical

Mitidieri, V. \& Ottone, N. E. Intrahepatic biliary ducts. Anatomic and surgical classification after cholangiographic findings. Int. J. Morphol., 33(4):1427-35, 2015.

Mitidieri, V. C. \& Mitidieri, A. Anatomía Quirúrgica de la Vía Biliar. En: Galindo, F. (Ed.). Enciclopedia Cirugía Digestiga. Buenos Aires, Sociedad Argentina de Cirugía Digestiva, 2017. Disponible en: http:// www.sacd.org.ar/437\%20Via\%20biliar\%20anat2017.pdf

Moore, K. L.; Dalley II, A. F. \& Agur, A. M. R. Clinically Oriented Anatomy. $6^{\text {th }}$ ed. Philadelphia, Lippincott Williams and Wilkins, 2009.

Panagouli, E. \& Venieratos, D. Right accessory hepatic artery arising from the left gastric artery: a case report. Rom. J. Morphol. Embryol., 52(3 Suppl.):1143-5, 2011.

Reichert, P. R.; Renz, J. F.; D'Albuquerque, L. A.; Rosenthal, P.; Lim, R. C.; Roberts, J. P.; Ascher, N. L. \& Emond, J. C. Surgical anatomy of the left lateral segment as applied to living-donor and split-liver transplantation: a clinicopathologic study. Ann. Surg., 232(5):658-64, 2000.

Sano, K.; Makuuchi, M. \& Sugawara, Y. Hepatic vein path and drainage area from the viewpoint of partial liver transplant. Biliary Tract Pancreas, 24(2):119-24, 2003.

Sinnatamby, C. S. Last's Anatomy: Regional and Applied. $10^{\text {th }}$ ed. Barcelona, Paidotribo, 2003.

Standring, S. Gray's Anatomy: The Anatomical Basis of Clinical Practice. $40^{\text {th }}$ ed. Edinburgh, Churchill Livingstone/Elsevier, 2008.

Tani, K.; Shindoh, J.; Akamatsu, N.; Arita, J.; Kaneko, J.; Sakamoto, Y.; Hasegawa, K. \& Kokudo, N. Venous drainage map of the liver for complex hepatobiliary surgery and liver transplantation. $H P B$ (Oxford), 18(12):1031-8, 2016.

Dirección para correspondencia

César Augusto Durand López

Cirujano del Hospital de Emergencias "José Casimiro Ulloa"

Hospital Emergencias

Av. Roosevelt 6355

Miraflores

Universidad Ricardo Palma

Lima

PERÚ

Email: cesaraugustoo1@gmail.com

Recibido : 24-07-2017

Aceptado: $20-10-2017$ 